\title{
A Simple Gravimetric Method to Determine Barometer Corrections
}

\author{
Randall M. Schoonover* \\ National Bureau of Standards, Washington, D.C. 20234
}

\begin{abstract}
April 23, 1980
Presented here is a gravimetric method to calibrate barometers. The difference in forces exerted on the pan of a balance is observed for two well characterized artifacts of nearly equal masses but different volumes. During the weighing, air temperature and relative humidity are measured; the ambient pressure is then calculated from an air density equation. A barometer correction is derived and then compared to an independent value based on a standard barometer. The data indicate that pressure can be calculated with an uncertainty (1 S.D.) of not more than $400 \mathrm{ppm}$ at one atmosphere.
\end{abstract}

Key words: Air density; air density equation; barometer calibration; barometric pressure; displacement volume; gravimetric method; mass artifacts; weighing.

\section{Introduction}

Many laboratories have modern commercial balances capable of near state-of-the-art weighing precision. Some laboratories, however, are not equipped to perform the necessary calibration of their instruments for measuring temperature, pressure and relative humidity, and hence systematic errors in the mass value assignment result.

Simple reliable instruments for measuring temperature and relative humidity are portable and can be sent elsewhere for calibration. This is not, however, generally true of the frequently-encountered aneroid and Fortin type barometers where accuracy to a few tenths of a millimeter of mercury is desirable. These instruments are best calibrated in place by pressure-defining instruments such as a standard mercury barometer or a dead-weight piston gage.

Presented here is a simple gravimetric method for a calibration of the barometer in the user's laboratory that does not require pressure equipment. Two well-characterized weights of grossly different displacement volumes are used to measure the air density directly by mass comparison on the balance $[1] ;{ }^{1}$ in addition, the ambient temperature and relative humidity must be measured. From these data, using an air density equation [2], ambient pressure can be calculated and compared to the barometer reading made at the time of weighing. The barometer correction is derived from the barometer reading and the calculated pressure.

\footnotetext{
*Center for Absolute Physical Quantities, National Measurement Laboratory.
}

' Figures in brackets indicate literature references at the end of this paper.
Although the gravimetric determination of pressure is unusual the gravimetric method to determine air density was demonstrated by Baxter [3] in 1921. Recent absolute determinations of air density $[1,4]$ have shown that air density calculations based on the gas laws are accurate to $4 \times 10^{-4}$. The data presented here result from mass comparisons [5] at two differing altitudes using a recent formulation [2] for the density of air in the buoyancy correction. Analysis of the data presented here suggests that a barometer correction made by the gravimetric method is accurate to $0.3 \mathrm{~mm} \mathrm{Hg}$ $(40 \mathrm{~Pa})$ or better.

\section{The Method}

Given two artifact masses, $A$ and $B$, with displacement volumes $V_{A}$ and $V_{B}$, respectively, the indicated difference, $I$, when compared on a balance is

$$
I=\hat{A}-B+\varrho\left(V_{B}-V_{A}\right)
$$

where $\varrho$ is the density of air at the time of measurement.

In this work, $I$ results from a single double substitution weighing ${ }^{2}$ using a five-observation format.

$$
I=\frac{\left(0_{1}-0_{2}-0_{3}+0_{4}\right) \Delta}{\left(0_{3}-0_{2}+0_{4}-0_{5}\right)}
$$

The balance observations $0_{1}$ through $0_{s}$ correspond to five loads placed on the balance pan, from which $\mathrm{I}$ is calculated. The five loads are weight $A$, weight $B, B$ plus weight $\Delta, A+\Delta$ and $A$. The sensitivity weight, $\Delta$, calibrates the balance response in mass units and is chosen to be larger than $A-B$. The buoyant effect on $\Delta$ is negligible with respect to the measurement precision and has been omitted. 
Air density variations may cause $I$ to be undesirably large for a given balance. Such occurrences are accommodated by adding small trimming weights of known mass and volume to either $A$ or $B$ or both. Therefore, a more general expression for $I$ is desirable and given as

$$
I=A-B+\delta_{1}-\delta_{2}+\varrho\left(V_{B}-V_{A}+V_{2}-V_{1}\right)
$$

where $\delta_{1}$ and $\delta_{2}$ are the small trimming weights and $V_{1}$ and $V_{2}$ are their respective volumes. The above weights are assumed to be at the ambient air temperature; if the volumes have been determined at some other temperature a correction for thermal expansion ${ }^{3}$ must be applied.

Rewriting eq (2) in terms of the air density at the time of weighing, we have

$$
\varrho=\frac{B-A+\delta_{2}-\delta_{1}+I}{V_{B}-V_{A}+V_{2}-V_{1}}
$$

We can now insert the gravimetrically derived value of $\varrho$ into an air density equation and formulate an expression for the ambient pressure, $P$.

Equation(43) of [2], a restricted version of the general air density formulation, was manipulated to accommodate a variation in ambient conditions and to accommodate pocket calculator computation. ${ }^{4}$ The resultant expression for the pressure during the time of weighing is

$$
P=\frac{\varrho T Z}{0.0034836}+0.0037960 U e_{s}
$$

where $\varrho$ is the gravimetric value given by eq (3).

In the above equations, $T$ is air temperature in kelvins, $U$ is percent relative humidity, $P$ is barometric pressure in pascals, $e_{S}$ is the saturation vapor pressure of water, $Z$ is the compressibility factor for moist air; $\varrho$ and $\varrho^{\prime}$ are in $\mathrm{kg} \mathrm{m}^{-3}$.

For convenience, the equation for $e_{s}$ as given in [2] is reproduced here.

$$
e_{S}=1.7526 \times 10^{11} \varepsilon^{(-5315.56 / T)}
$$

$\varepsilon$ is the base of natural logarithms. References for $e_{s}$ in tabular form are given in [2].

${ }^{3}$ If the volume of a weight is given at temperature $t_{R}$ then the volume at any temperature $t$ is computed from the formula $V_{t}=V_{t R}\left[1+3 \alpha\left(t-t_{R}\right)\right]$. It is assumed that the thermal coefficient of expansion, $\alpha$, is known. The use of $3 \alpha$ for the coefficient of cubical expansion also assumes that the material is isotropic.

${ }^{4}$ The modified air density formula is

$$
\varrho^{\prime}=\frac{0.0034836}{T Z}\left(P-0.0037960 U \mathrm{e}_{s}\right)
$$

Q has been primed to indicate a calculated value of air density as opposed to the gravimetric value.
The sole change made to eq (43) was to put the compressibility term, $Z$, explicitly in the formula as a variable. Doing so improves the accuracy at laboratories whose elevation and ambient conditions differ from those applicable to the NBS. The remaining restriction, the assumed $\mathrm{CO}_{2}$ level, still applies. As a convenience to the reader, a table of $Z$ values from [2] is reproduced in appendix II of this report.

In summary, if one weighs two artifacts of nearly equal mass but with a large volume difference and records the ambient temperature, relative humidity and masses and displacement volumes of the trimming weights, if required, ambient pressure can be calculated and a barometer correction derived.

\section{Artifacts and Instrumentation}

The temperature and relative humidity were respectively measured with a mercury-in-glass thermometer and an electric hygrometer. The calibration and accuracy of these devices, as well as the certification of masses and volumes of the artifacts and trimming weights are adequately described in [5]. However, to aid in performing this method of barometer calibration, additional details concerning the artifacts and trimming weights are given in appendix I of this report.

\section{Test Data}

The artifacts, the masses of which are designated $A$ and $B$ in eq (1), correspond to a ring-shaped weight and a hermetically-sealed hollow cylindrical weight of significantly larger volume. There are two such pairs of weights, referred to as Pair 1 and Pair 2. The weights of Pair 1 are referred to as $R_{1}$ and $H_{1}$ and of Pair 2 as $R_{2}$ and $H_{2}$, where $R$ designates the ring weight and $H$ the hollow weight for each pair.

The weighings were performed at NBS Washington, 150 $m$ elevation, and at the Sandia Laboratory in Albuquerque, N.M. located at $1600 \mathrm{~m}$ elevation. In some instances the laboratory temperature was controlled whereas the pressure was always free to vary with the prevailing weather conditions. A time period of several months elapsed from the first to the last measurement. For each day of measurement the artifact pairs were weighed only once, however both pairs were not always weighed on the same day. In all there were 22 artifact weighings with one being discarded as an obvious outlier.

The measurements of each day began and usually ended with an independent calibration of the aneroid barometer in use by comparison with a standard mercury barometer. Two aneroid barometers were required to accommodate the pressure range encountered between 150 and $1600 \mathrm{~m}$ elevation with sufficient resolution and reproducibility; however, 
the same standard mercury barometer was used throughout the measurements to define pressure.

\section{Results}

The data as summarized in table 1 are segregated into two groups by altitude and the corresponding aneroid barometer. The column of gravimetrically derived values for air density indicates the magnitude and the daily variations encountered at each location. For convenience to the reader all pressures are given in both pascals $(\mathrm{Pa})$ and millimeters of mercury ( $\mathrm{mm} \mathrm{Hg}$ ). The aneroid barometer scales are graduated in $\mathrm{mm} \mathrm{Hg}$ and their corrections are likewise given. The insert at the bottom of table $\mathrm{I}$ is a complete data set for run 22 .

The mean correction, $\bar{X}$, and the estimate of the standard

TABLE 1. These data summarize twenty-two aneroid barometer calibrations achieved by the gravimetric method. Also given are the results of an independent comparison with a standard mercury barometer.

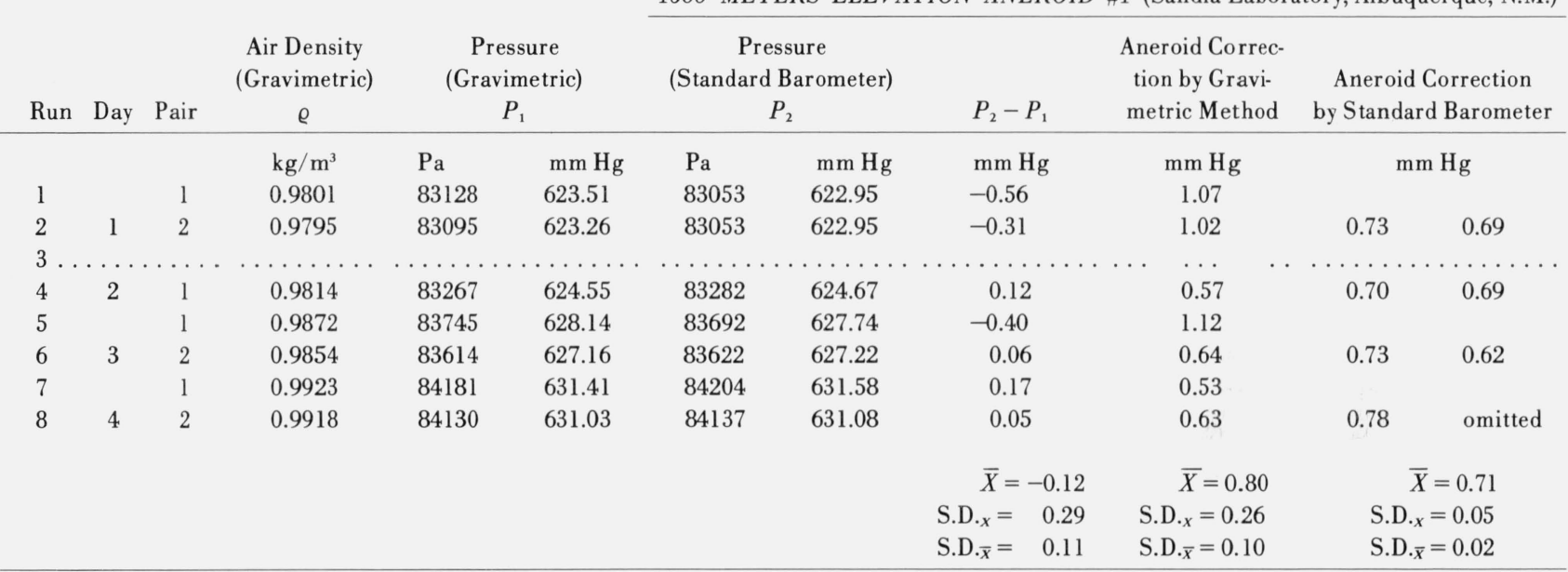

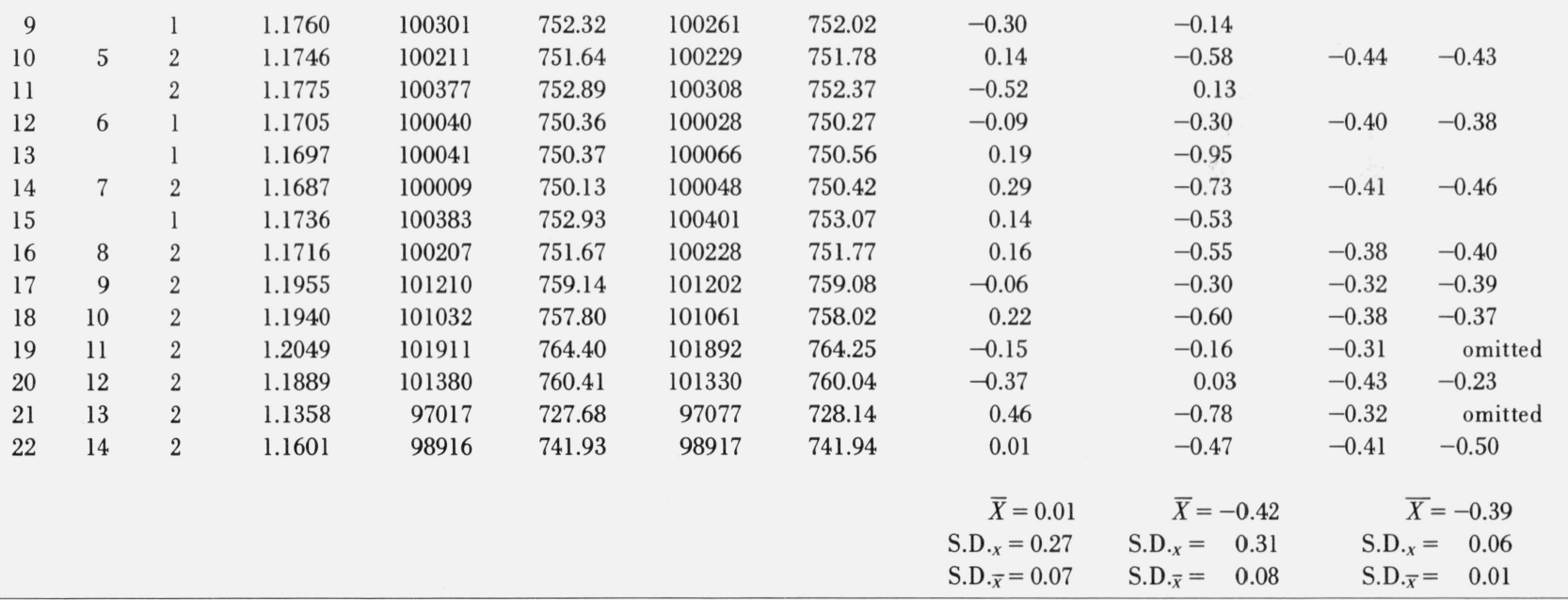

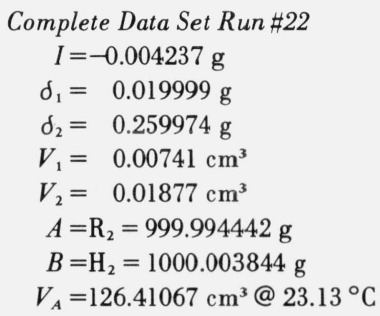

${ }^{a} \mathrm{~kg} / \mathrm{m}^{3}$ are required for use in eq $(2)$
150 METERS ELEVATION ANEROID \#2 (N.B.S. Laboratory, Gaithersburg, MD.)

$$
\begin{aligned}
V_{B} & =337.71691 \mathrm{~cm}^{3} @ 23.13{ }^{\circ} \mathrm{C} \\
T & =296.28 \mathrm{~K} \\
U & =26.6 \text { percent }
\end{aligned}
$$

Aneroid Barometer Observation $=741.94 \mathrm{~mm} \mathrm{Hg}$

$$
Z=0.99964
$$$$
e_{s}=2831 \mathrm{~Pa}
$$$$
\varrho=0.0011601 \mathrm{~g} / \mathrm{cm}^{3} a=1.1601 \mathrm{~kg} / \mathrm{m}^{3}
$$

$P_{2}=98917 \mathrm{~Pa}=741.94 \mathrm{~mm} \mathrm{Hg}$ 
deviation of the mean, S.D. $\bar{x}$, are given for the gravimetrically-dervied aneroid barometer corrections and those assigned by calibration with the standard barometer. Also given, for both methods of calibration, is the estimate of the standard deviation for a single observation, S.D.x. Similar estimates are given for the gravimetrically derived pressure, $P_{1}$, and that assigned by the standard barometer, $P_{2}$.

One would expect the difference between $P_{1}$ and $P_{2}$ to be near zero. It is not surprising to find the difference in mean values $\left(P_{2}-P_{1}\right)$ smaller at the lower elevation site as weighing conditions were somewhat better than those of the higher elevation where air currents and vibration were detrimental. This contention is supported by the pooled estimates of balance standard deviation, $60 \mu \mathrm{g}$ for the higher laboratory and $40 \mu \mathrm{g}$ for the lower one. Futhermore, the corrections assigned to the aneroid barometers by the standard barometer at both locations do not significantly differ in precision and therefore make the same error contribution at both locales, leaving only errors associated with the weighing procedure to account for the difference. However, the non-zero difference is well within l S.D. for the gravimetric method and is not statistically significant.

The above discussion holds true for the resultant aneroid barometer corrections.

The barometric pressure $(760 \mathrm{~mm} \mathrm{Hg})$ as indicated by the standard barometer is estimated to be in error by 1 part in $30000,0.025 \mathrm{~mm} \mathrm{Hg}$, and is dominated by reading error that is random in nature. The scale readings of the aneroid barometers are estimated to have an uncertainty of $1 / 10$ of a division, $0.02 \mathrm{~mm} \mathrm{Hg}$. In addition, atmospheric pressure variations that occur between calibrations expose the inherent non-reproducibility of the aneroid barometer. The resultant errors are decreased by the number of repeated calibrations and are reflected by S.D. $\vec{x}$ for the aneroid calibrations based on the standard barometer. The random uncertainty of the mean correction, $\bar{X}$, for the aneroid barometer as derived by comparison with the standard barometer, is taken to be S.D. $\bar{x}$.

Uncertainty in the barometric pressure as determined gravimetrically arises from errors in the characterization of mass and volume of the artifacts, weighing errors (including the lack of thermal equilibrium) and errors associated with the parameters in the equation for barometric pressure. Again, random errors dominate and the inconsequential known systematic errors have been omitted here.

A relative uncertainty of $290 \mathrm{ppm}$, at the level of 1 S.D., is assigned to the gravimetrically determined air density and is combined, by the method of root sum squares, with 210 ppm uncertainty (at 1 S.D.) resulting from the equation for pressure. The resultant estimated uncertainty in the gravimetrically determined pressure is $360 \mathrm{ppm}$. For barometric pressures near $760 \mathrm{~mm} \mathrm{Hg}$ this amounts to $0.27 \mathrm{~mm} \mathrm{Hg}$ and to $0.23 \mathrm{~mm} \mathrm{Hg}$ for pressures near $630 \mathrm{~mm} \mathrm{Hg}$.

\section{Conclusions}

The relative uncertainty (at 1 S.D.) in the aneroid barometer correction has been shown to be $360 \mathrm{ppm}$. This uncertainty will be propagated directly to barometric pressure determined by the instruments in combination with the random reading uncertainty of $0.02 \mathrm{~mm} \mathrm{Hg}$. The latter is usually diminished by making repeated observations of pressure during the weighing operation.

It is suggested that the gravimetric barometer calibration will adequately serve the needs of many laboratories for measurements such as precision weighing, within the limits discussed here. ${ }^{5}$ Of course, the calibration should be performed several times and preferably on different days with significant variation in ambient pressure.

The artifacts used in this work have characteristics nearly as close to ideal as good practice allows. Experience [5] indicates that the hermetically-sealed hollow weight made from stainless steel can be replaced with a cylinder made from an aluminum alloy. Furthermore, in the author's opinion, the ring weight of nearly equal surface area could be fabricated from brass tubing, with the added benefit of having a thermal conductivity similar to that of aluminum.

This latter property may be of importance although it has not been investigated here. Similar reflective properties could be achieved by gold plating both artifacts. Under no circumstances should the aluminum weight be anodized, as anodized surfaces may become electrically charged and cause serious weighing errors.

The author wishes to thank Harry R. Johnson, formerly of the NBS Office of Weights and Measures, who first introduced the barometer calibration problem to him, and to apologize for the delay in demonstrating that the suggested solution was valid. The author is deeply indebted to Horace A. Bowman who anticipated surface-dependent effects and designed the artifacts to minimize the problem. Thanks are due to C. Leon Carroll who took great care in making a close mass adjustment for the artifacts and in polishing their surfaces to their highly specular finish and took part in their volume determination. Special thanks are due Frank E. Jones for being patient and free with advice on the modification of the air density equation and to Richard S. Davis who gave valuable consultation and statistical help.

\section{References}

[1] Bowman, H. A. and Schoonover, R. M. with Appendix by Mildred W. Jones, Procedure for High Precision Density Determinations by Hydrostatic Weighing, J. Res. Nat. Bur. Stand. (U.S.) 71C, Engr. and Instr.), No. 3, 179-198, (July-Aug. 1967).

${ }^{s}$ The resultant barometer calibration is not traceable to the N.B.S. pressure standards. 
[2] Jones, F. E., The Air Density Equation and the Transfer of the Mass Unit, J. Res. Nat. Bur. Stand. (U.S.) 83, No. 5, 419-428, Sept.-Oct. 1978).

[3] Baxter, G. P., J. Am. Chem. Soc. 43, 1317-1318 (1921).

[4] Koch, W. F., Davis, R.S. and Bower, V. E., Direct Determination of Air Density in a Balance Through Artifacts Characterized in an Evacuated Weighing Chamber, J. Res. Nat. Bur. Stand. (U.S.) 83, No. 5, 407-413 (Sept.-Oct. 1978).

[5] Schoonover, R. M., Davis, R. S., Driver, R. G. and Bower, V. E., A Practical Test of the Air Density Equation in Standards Laboratories at Differing Altitude, J. Res. Nat. Bur. Stand. (U.S.) 85, No. 1, 27-38 (Jan.-Feb. 1980).

\section{Appendix I}

\subsection{Equipment}

Fabrication of the artifacts is directed toward optimizing the difference in displacement volume between them and to adjust their masses close to $1 \mathrm{~kg}$, a precision balance capacity available to most laboratories. There are other practical aspects that also must be considered, however, such as the volume measurement, fabrication techniques and surfacedependent effects related to the lack of thermal equilibrium [5] during the weighing procedure.

The artifacts used here [5] were designed to minimize surface dependent effects and to have a nominal mass of $1 \mathrm{~kg}$ with densities of about $2.7 \mathrm{~g} \mathrm{~cm}^{-33}$ for one and $7.8 \mathrm{~g} \mathrm{~cm}^{-3}$ for the other, and nearly equal surface areas and finish. The surface area of the less dense weight is minimized in the usual manner by designing a cylinder of equal height and diameter. Since both artifacts are of the same material, stainless steel, the low density weight is a sealed hollow cylinder of substantial wall thickness and the other is in the shape of a tube. One is tempted, by the physical principles, to fabricate the hollow cylinder of an even lower density material; however, doing so unnecessarily complicates the hydrostatic weighing for the determination of volume by approaching hydrostatic fluid density i.e. the positive buoyancy point. In addition, the wall thickness of the cylinder may not be substantial, resulting in unnecessary fragility and perhaps compressibility.

Although it is the difference in mass between the artifacts that is of interest here, derivation of mass is by assignment using well-known intercomparison techniques with standards of mass [5]. This method has many statistical advantages over a simple difference measurement. A mass of $1 \mathrm{~kg}$ is a convenience to both the mass assignment and the hydrostatic weighing procedures used to determine the displacement volume.

\subsection{Trimming Weights}

The trimming weights are commercially available and are made from sheets of known alloys. This permits the use of a handbook value for their densities in computing displacement volume. It is a routine matter to assign mass values accurate to a few micrograms in the range of $500 \mathrm{mg}$ to 1 mg. The amount of trimming weights used in the barometer calibration is small provided the artifacts are adjusted close to each other and to the nominal value of the mass standard. Failure to meet these criteria may require measuring the volume of the trimming weights in place of a simple estimate based on alloy density.

\section{Appendix II}

TABLE 2. Compressibility factor, $\mathrm{Z}$, for $\mathrm{CO}_{2}$-free air

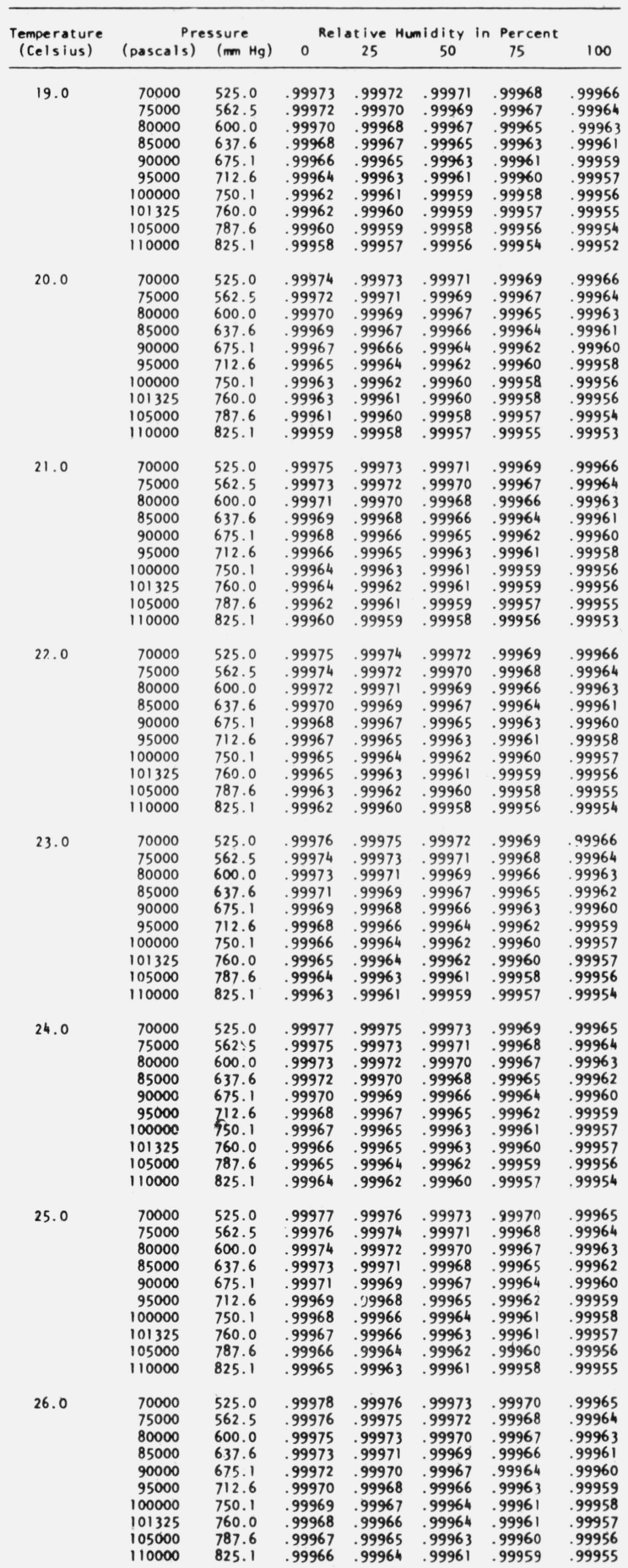


